\title{
Effects of KRAS Gene Mutations in Gynaecological Malignancies
}

\author{
Keerthana $\mathbf{S}^{1}$, Akram Husain $\mathrm{RS}^{2}$ and Ramakrishnan $\mathrm{V}^{2 *}$ \\ ${ }^{1}$ Medical Genetics and Molecular Diagnostics, Faculty of Allied Health Sciences, India \\ ${ }^{2}$ Genetics Lab, Faculty of Allied Health Sciences, India \\ *Corresponding author: Ramakrishnan V, Faculty of Allied Health Sciences, Chettinad Academy of Research and Education, Chettinad Health City, \\ Kelambakkam - 603 103, India
}

Submission: 眥January 18, 2018; Published: 無 May 15, 2018

\begin{abstract}
Gynaecological disorders and malignancies affect the female reproductive system. Every woman in her life time suffers from gynaecological problem at some extent. The development and pathogenesis of gynaecological disorders is still remains unclear, several clinical and epidemiological studies have documented that they can be triggered by environmental, life-style, genetic factors and epigenetic alterations.The KRAS (Kristen rat sarcoma viral oncogene) gene is the commonly mutated oncogene. This gene provides instructions for making a protein called as K-Ras, which plays a vital role in cell division, differentiation and apoptosis. The frequency of mutations in this oncogene is highly reported in gynaecological cancers. This review highlights the common mutations observed in KRAS gene that is associated with breast, cervical, ovarian and endometrial cancer. These genetic variations can be considered with their clinical significance and might be used as diagnostic biomarkers and testing for RAS pathway mutations.
\end{abstract}

Keywords: KRAS; Mutation; Gynaecological malignancies; Breast cancer; Cervical cancer

\section{Introduction}

Cancer is a complex collection of diseases that interfere with regular cellular growth and death; specific molecular alterations induce a normal cell to become malignant, have the ability to invade and metastasize from the primary lesion, but the spectrum of these alterations vary substantially among cancers [1]. Gynaecological malignancies are the most common cancers in women community and hence it's an important alarming public health issue. Due to lack of awareness of this disease, pathological status and scarce of screening facilities in developing countries lead most women to report at advanced stages, adversely affecting the prognosis and clinical outcomes [2]. The risk factors of cancer in women include, family history of ovarian cancer or cancers of the uterus, breast, beyond the age of menopause, obese, have never been pregnant or had infertility problems and the usage of medication tamoxifen or use unopposed oestrogen [3]. Recently, Genome Wide Association Studies (GWAS) in major gynaecological malignancies have revealed susceptible genes such as RAD51C, TP53, BRCA1, BRCA2, CHEK2, BRIP1, FAT10, MLH1, KRAS, PIK3CA and PTEN associated with the disease pathogenesis [4]. This review focuses on the reported genetic mutations occurring in Kirsten Rat Sarcoma Viral Oncogene (KRAS) of cervical, ovarian, endometrial and breast cancer respectively.

\section{KRAS Gene}

KRAS is a Protein Coding Proto-Oncogene and there are two copies of the KRAS gene in the human genome, designated KRAS1 and KRAS2. It is located in the $12^{\text {th }}$ chromosome spanning with 6 exons [5]. Figure 1 explains the chromosomal location, exon structure, position of reported mutations at their corresponding genomic positions and their protein domains. The KRAS gene which provides instructions for making a protein called K-Ras which belongs to small GTPase super family and Ras family. They mainly function in signal transduction pathways, RAS/MAPK pathway and limited to cell membranes consisting of an isoprenyl group on its C-terminus [6]. The K-Ras protein is a GTPase, converts a GTP molecule into GDP molecule that plays a major role in protein biosynthesis, single transduction, differentiation and translocation. The K-Ras protein acts like a molecular switch that is turned on and off by the GTP, GDP molecules [7]. It is the most frequently mutated oncogene in cancer and associated with poor diagnosis, resistance to treatment and therapy [8].

\section{Gynaecological Cancers}

The development of cancer is a multiple step process, characterized by the accumulation of environmental, life-style, 


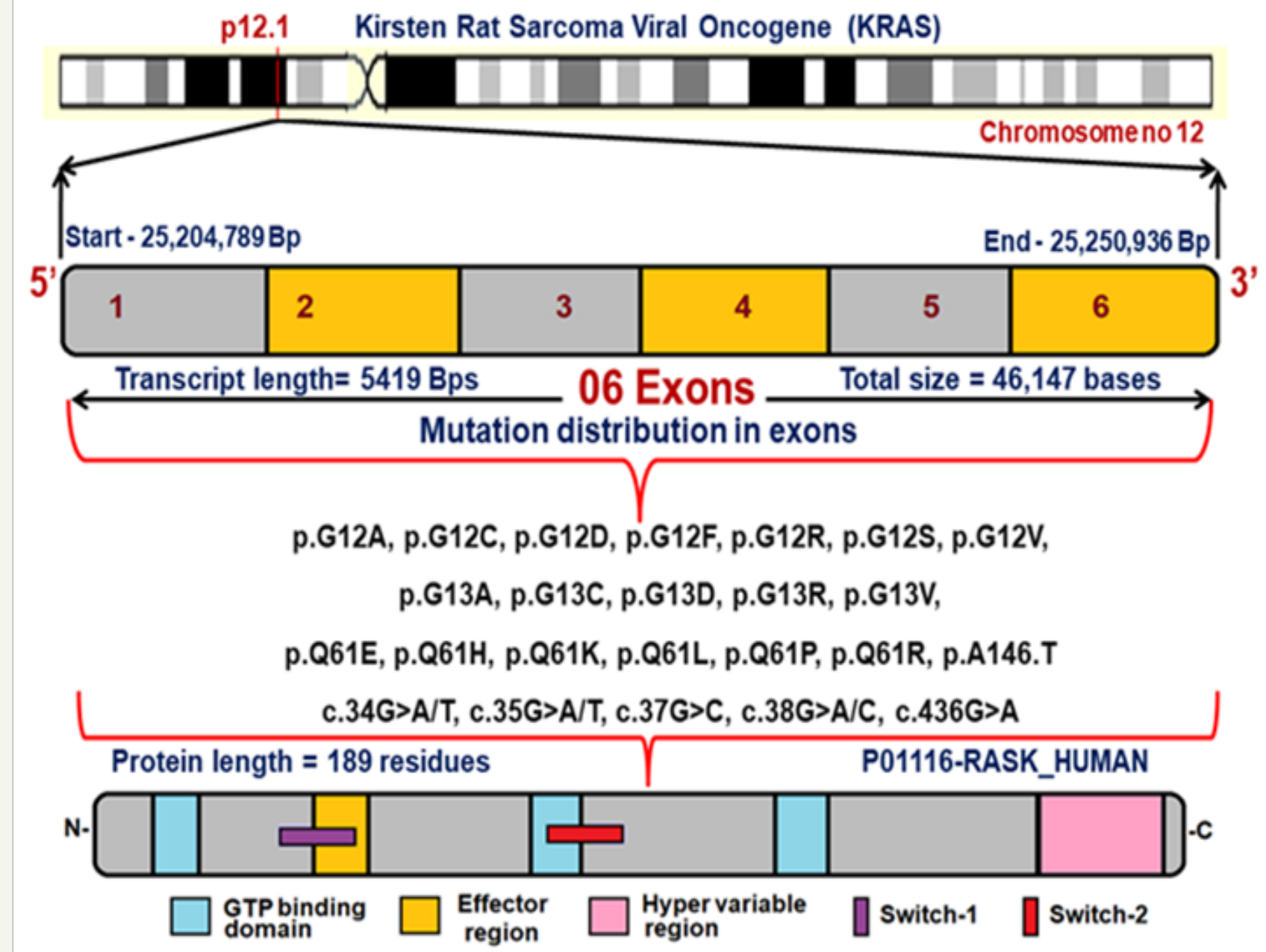

Figure 1: Exon structure of KRAS gene with reported mutations and their protein domains.

\section{KRAS Gene Mutation in Cervical Cancer}

Cervical cancer is one of the leading causes for female death globally. It is the second most common gynaecologic malignancy and constitute for significant public health problem in women community [9]. The incidence of adenocarcinoma of the cervix has risen from 5 to $24 \%$ [10]. Several studies have demonstrated that adenocarcinoma confers worse prognosis with higher rates of lymph node involvement and distant metastases with decreased survival across stages compared with squamous cell carcinoma [11]. Human Papilloma Virus (HPV) is sexually transmitted and one of the prime etiological agent in the development of cervical cancer [12]. In addition, KRAS mutations have been identified widely in cervical adenocarcinomas and most of them were missense mutations of codon G12; these are well described by activating mutations that have found to be associated with poor prognosis in metastasis with resistance to EGFR targeted therapies in various cancers [13]. The common mutations seen in KRAS gene are G>A transition, G12D,
c.35G-A, G>T transversion, C12GGT>TGT, C12GGC>GAC, GGT>TTT, $G G T>T G T, G G T>G T T$ [14]. Kras genetic variations are an active area of research with promising pre-clinical data and research is focussed on mitogen-activated protein/extracellular signal-regulated kinase (MEK) inhibitors [15].

\section{KRAS Gene Mutation in Ovarian Cancer}

Ovarian cancer (OC) is the seventh most commonly diagnosed malignancy among women worldwide [16]. According to global estimates 239,000 new cases were detected each year and 152,000 people annually die from the disease [17]. The origin and pathogenesis of $\mathrm{OC}$ is not well understood and, most tumours appear to be originated from other gynaecological tissues and lead to the ovary secondarily. Figure 2 illustrates the incidence age-standardized rate of ovarian cancer worldwide. This data was retrieved from the International Agency for Research on Cancer (IARC) GLOBOCAN 2012 database. 


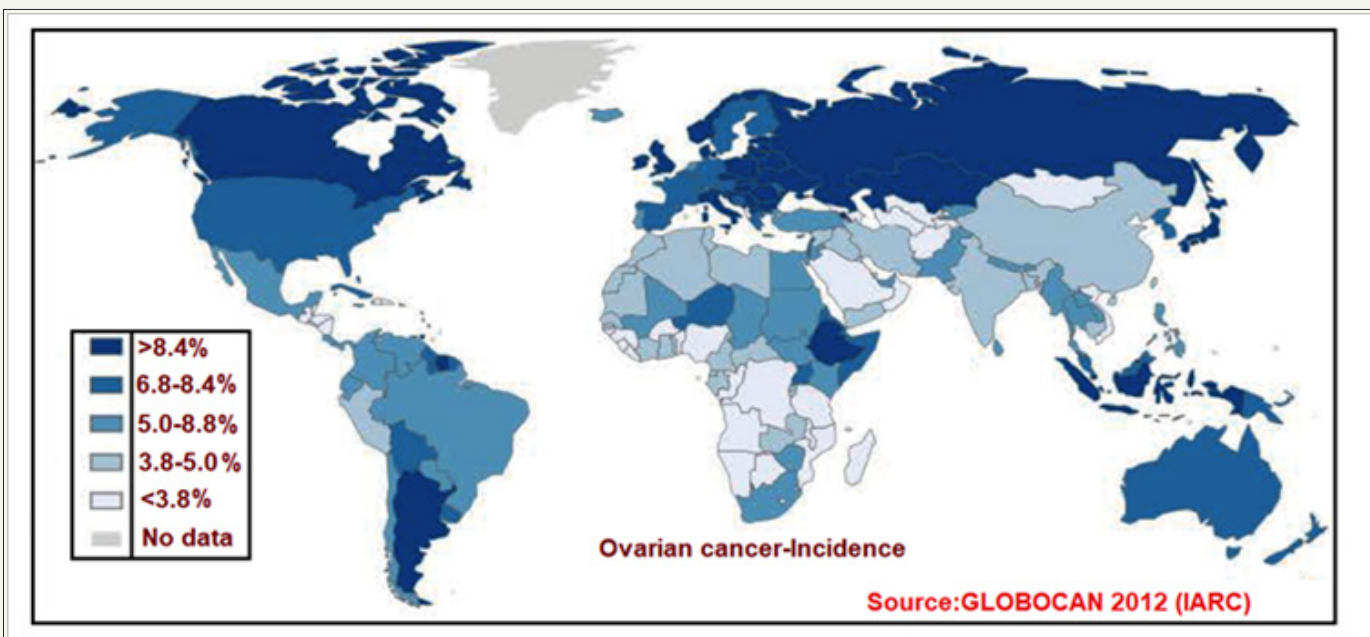

Figure 2: World map showing incidence (Age-standardized rate) of ovarian cancer.

Morphological, clinical and genetic studies have documented several hypotheses of origination; mainly for high-grade serous tumours that lack a clear progression model [18]. Numerous clinical studies have revealed the presence of mutations at codon 12 of the KRAS gene in particular association with ovarian cancer. The frequency of G12 mutations was $6.2 \%$ of ovarian cancer tissue and $14.3 \%$ in the borderline ovarian cancer [19]. The genetic variations in KRAS gene in ovarian carcinoma is consistent with the similar finding in melanoma and reveals us that KRAS mutation has equivalent effect on tumorigenesis [20].

\section{KRAS Gene Mutation in Endometrial Cancer}

Endometrial cancer is the most common pelvic gynaecological malignancy in developed nations. Endometrium is a complex tissue that lines in the uterine cavity and is composed of both glandular and stromal elements [21]. The number of new cases of endometrial cancer was $25.7 / 100,000$ women and the mortality rate was 4.6/100,000 women per year in worldwide [22]. The cyclic proliferation, differentiation of both components is controlled and coordinated by steroid hormones produced by the ovary. In general, oestrogen promotes for the proliferation and progesterone leads to the differentiation of both components [23]. Endometrial carcinoma arises from the epithelial component and has been thought of as a single disease. However, clinico-pathological studies suggested that endometrial carcinoma could be broadly divided into two major types, referred to as type- 1 and type-2 [24]. The most commonly altered oncogene in endometrial carcinoma is KRAS, which is mutated in $10-30 \%$ of cases. The mutations have been found in all grades of endometrial carcinoma and have been reported in complex atypical hyperplasia, suggesting a role for KRAS mutation in this tumour type [25].

\section{KRAS Gene Mutation in Breast Cancer}

Breast cancer is the most frequently diagnosed gynaecological malignancy in women and ranks second among causes for cancer related mortality in women community [26]. The incidence rates vary from 19.3/100,000 worldwide and the development of breast cancer is complicated processes involving multiple factors and results from a series of genetic alterations [27]. Breast cancer is inherited and women with first-degree relative diagnosed to have breast cancer posses increase risk. The candidate genes such as BRCA1, BRCA2 harbours high frequency of mutation followed by other genes such as KRAS [28]. A study on mutational analysis in human breast cancer cell lines has revealed $18 \%$ of association with KRAS gene mutation and most of the KRAS genetic variations were localized on $12^{\text {th }}$ codon and lesser at 61st codon [29]. KRAS mutations have also been reported to be the risk factor for triple negative breast cancer in premenopausal women [30].

\section{Conclusion}

In current scenario, it is important to emphasize the risk factors of gynaecological malignancies apart from highly penetrant and common genetic mutations. This review highlights the necessity to focus on KRAS gene variations, their effect on protein structure and function. Finally, these variants might be used in early diagnosis of cancer, prognosis of the disease followed by therapeutic measures so that the women community gets protected from various types of malignancies.

\section{Conflicts of interest}

There are no conflicts of interest.

\section{References}

1. Hanahan D, Weinberg RA (2011) Hallmarks of cancer: the next generation. Cell 144(5): 646-674.

2. Anderson BO, Yip CH, Smith RA, Shyyan R, Sener SF, et al. (2008) Guideline implementation for breast healthcare in low income and middle-income countries. Cancer 113(S8): 2221-2243.

3. Ayhan A, Salman MC, Celik H, Dursun P, Ozyuncu O, et al. (2004) Association between fertility drugs and gynecologic cancers, breast cancer, and childhood cancers. Acta Obstet Gynecol Scand 83(12): 11041111.

4. Hardy J, Singleton A (2009) Genomewide association studies and human disease. N Engl J Med 360(17): 1759-1768. 
5. Flicek P, Ahmed I, Amode MR, Barrell D, Beal K, et al. (2012) Ensembl 2013. Nucleic Acids Res 41(D1): D48-55.

6. Buday L, Downward J (2008) Many faces of Ras activation. Biochim Biophys Acta 1786(2): 178-187.

7. Scita G, Tenca P, Frittoli E, Tocchetti A, Innocenti M, et al. (2000) Signaling from Ras to Rac and beyond: not just a matter of GEFs. The EMBO Journal 19(11): 2393-2328.

8. Ciocca DR, Calderwood SK (2005) Heat shock proteins in cancer: diagnostic, prognostic, predictive, and treatment implications. Cell Stress Chaperones 10(2): 86-103.

9. Husain RA, Ramakrishnan V (2015) Global variation of human papillomavirus genotypes and selected genes involved in cervical malignancies. Ann Glob Health 81(5): 675-683.

10. Kumar N (2016) Cervical cancer; a nightmare for womanhood: review of recent advances. Women's Health \& Gynaecology 9: 30-34.

11. Allen DC (2013) Malignant melanoma. In Histopathology Reporting, Springer, London.

12. Akram Husain RS, Ramakrishnan V (2016) A review of risk factors in the development of cervical malignancy. Cancer Sci Res Open Access 3(1): $1-4$.

13. Chong CR, Jänne PA (2013) The quest to overcome resistance to EGFRtargeted therapies in cancer. Nature Medicine 19(11): 1389-1400.

14. Jančík S, Drábek J, Radzioch D, Hajdúch M (2010) Clinical relevance of KRAS in human cancers. Bio Med Research International 18: 201-210.

15. Roberts PJ, Der CJ (2007) Targeting the Raf-MEK-ERK mitogen-activated protein kinase cascade for the treatment of cancer. Oncogene 26(22): 3291-3310.

16. Reid BM, Permuth JB, Sellers TA (2017) Epidemiology of ovarian cancer: a review. Cancer Biology \& Medicine 14(1): 9.

17. Ferlay J, Soerjomataram I, Ervik M, Dikshit R, GLOBOCAN 2012 v1.0, Cancer Incidence and Mortality
18. http://globocan.iarc.fr

19. Prat J (2012) Ovarian carcinomas: five distinct diseases with different origins, genetic alterations, and clinicopathological features. Virchows Archiv 460(3): 237-249.

20. Shih IM, Kurman RJ (2004) Ovarian tumorigenesis: a proposed model based on morphological and molecular genetic analysis. Am J Pathol 164(5): 1511-1518.

21. Ellenson LH, Wu TC (2004) Focus on endometrial and cervical cancer. Cancer Cell 5(6): 533-538.

22. Basen-Engquist K, Chang M (2011) Obesity and cancer risk: recent review and evidence. Curr Oncol Rep 13(1): 71-76.

23. Wallace AE, Gibson DA, Saunders PT, Jabbour HN (2010) Inflammatory events in endometrial adenocarcinoma. Journal of Endocrinology 206(2): 141-157.

24. Felix AS, Weissfeld JL, Stone RA, Bowser R, Chivukula M, et al. (2010) Factors associated with Type I and Type II endometrial cancer. Cancer Causes \& Control 21(11): 1851-1856.

25. Lax SF (2003) Molecular genetic pathways in various types of endometrial carcinoma: from a phenotypical to a molecular-based classification. Virchows Archiv 444(3): 213-223

26. Pecorelli S, Favalli G, Zigliani L, Odicino F (20003) Cancer in women. IInt J Gynaecol Obstet 82(3): 369-379.

27. Jones PA, Baylin SB (2007) The epigenomics of cancer. Cell 128(4): 683692.

28. Vogelstein B, Papadopoulos N, Velculescu VE, Zhou S, Diaz LA, et al. (2013) Cancer genome landscapes. Science 339(6127): 1546-1558.

29. Wood LD, Parsons DW, Jones S, Lin J, Sjöblom T, (2007) The genomic landscapes of human breast and colorectal cancers. Science 318(5853): 1108-1103.

30. Yamamoto Y, Iwase H (2010) Clinicopathological features and treatment strategy for triple-negative breast cancer. Int J Clin Oncol 2010 15(4): 341-351.
Creative Commons Attribution 4.0 International License

For possible submissions Click Here

\section{Submit Article}

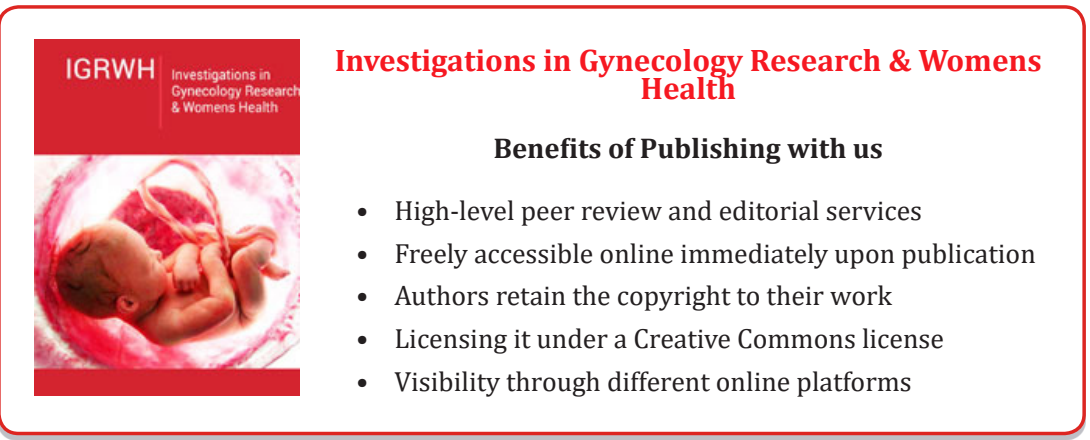

